\title{
Deviations of the Light Distribution from the Sersic's model in Elliptical galaxies
}

\section{Dr. Saad Mohmood Younis Omar Basil Mohammad Saleh Department of Physics / College of Education University of Mosul}

Received
$17 / 05 / 2010$
Accepted

$03 / 11 / 2010$

\begin{abstract}
(الملخص:

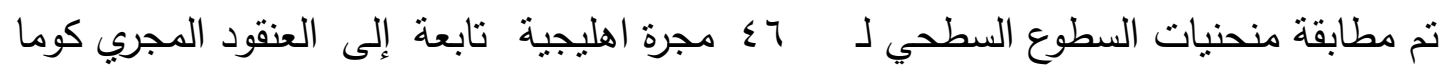
باستخدام نموذج سيرسك المعمم الانحرافات >

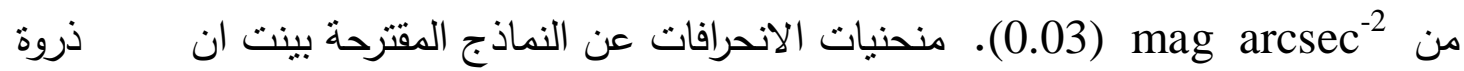
الانحرافات السالبة لجميع المجرات وجدت في حدود المسافة القطبية المختزلة به. . . ، وبينما كانت ذروة الانحرافات الموجبة للماذج المعتمدة وجدت في حدود المسافة القطبية المختزلة

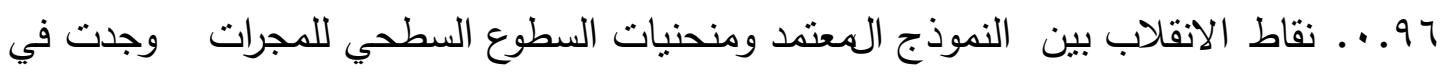
حدود المسافة القطبية المختزلة 0.65، 0.83 و 1.0.
\end{abstract}

\section{Abstract:}

The surface brightness profiles of 46 elliptical galaxies, belong to Coma Cluster of galaxies have been fitted by the generalized Sersic $\mathrm{r}^{1 / n}-$ law for the intermediate radius range $0.1 \leq\left(\frac{x}{x_{e}}\right) \leq 1.5$. The mean deviations $<\delta \mu>$ between the adopted model and the surface brightness profiles of the galaxies found to be less than $(0.03) \mathrm{mag} \operatorname{arcsec}^{-2}$. The deviation profiles between the observations and the adopted models show that the maximum negative deviation found to be around the reduced radius of 0.86 , while the maximum positive deviation were found to be around the reduced radius of 0.96 . The crossing points between the adopted models and the observed surface brightness profiles were found to be around the reduced radius $0.65,0.83$ and 1.0. 


\section{Introduction:}

The surface brightness profiles of elliptical galaxies are often fitted by the empirical $\mathrm{r}^{1 / 4}$ - law: (de Vauouleurs, 1948)

$$
\mu(R)=\mu_{e}+8.3275\left[\left(\frac{R}{R_{e}}\right)^{1 / 4}-1\right]
$$

Where $\mu(R)$ is the surface brightness (magnitude per $\operatorname{arcsec}^{2}$ ), $\mathrm{R}_{\mathrm{e}}$ is the radial distance( $\operatorname{arcsec}), R_{e}$ represents the effective radius (arcsec) which determine the isophote that contains half the total light, $\mu_{e}$ is the surface brightness at the effective radius. The $\mathrm{r}^{1 / 4}-$ law provides a good description for surface brightness at intermediate region of the elliptical galaxies (Okamura 1988), also describe the light distribution in lenticular galaxies (SO), and the bulges of spiral galaxies (Capaceioli etal 1993). Recently, the empirical $r^{1 / 4}$ - law has been replaced by the generalized $r^{1 / n}-$ law, originally proposed by Sersic (1968), which is given by the formula:

$$
\mu(R)=\mu_{e}+1.0857 b_{n}\left[\left(\frac{R}{R_{e}}\right)^{1 / n}-1\right]
$$

The coefficient $b_{n}$ is a function of the shape parameter $n$, which can be chosen in such a way that the scale-radius $R_{e}$ encloses half the total luminosity, a good approximation is $b_{n} \approx 2 n-0.327$ for $1 \leq n \leq 15$ (Trujillo et al., 2001). As the $\mathrm{r}^{1 / \mathrm{n}}$ - law has an additional new free parameter (n) besides the effective $R_{e}$, this law fit very well the surface brightness profiles for elliptical and SO galaxies (Caon et al., 1993), this law found to be fit the dwarf galaxies too Cellone, (1999).

Burkert (1993) has studied the intermediate axis surface brightness distribution of a large samples of elliptical galaxies, He has shown that the $\mathrm{r}^{1 / 4}$ - law provides an excellent fit to the observed brightness distribution within the radius range $0.1 R_{e} \leq R \leq 1.5 R_{e}$, with mean deviations $<\delta \mu>$ smaller than $0.1 \mathrm{mag} \operatorname{arcsec}^{-2}$ and the maximum deviations smaller than $0.2 \mathrm{mag} \operatorname{arcsec}^{-2}$. Younis $(2000 \mathrm{a}, \mathrm{b})$ has studied the systematic deviation from the $\mathrm{r}^{1 / 4}$ - law for two samples of elliptical galaxies and he found that the mean deviation are less than $0.12 \mathrm{mag} \operatorname{arcsec}^{-2}$, for one sample and less than 0.09 mag. $\operatorname{arcsec}^{-2}$ for the other. In this paper we analyzed the surface brightness distribution of 46 elliptical galaxies belong to Coma Cluster of galaxies published by Jorgensen et al., (1992) with the model proposed by Sersic $\left(\mathrm{r}^{1 / \mathrm{n}}-\right.$ law $)$ for the radial range $0.1 \leq\left(\frac{R}{R_{e}}\right) \leq 1.5$.

\section{Data Reduction:}

In present work the Sersic law has been used to model the observed surface brightness profiles of the adopted sample of galaxies for the 
intermediate radial range $0.1 \leq\left(\frac{x}{x_{e}}\right) \leq 1.5$, this range cover more than $60 \%$ of the total luminous mass which provides a good representation of the distribution of the visible matter (Burket 1993).

The effective radius $\mathrm{x}_{\mathrm{e}}$ for the adopted radial range was determined in a self-consistent method. The first estimated value for the $\mathrm{x}_{\mathrm{e}}$ was derived from the $\mathrm{r}^{1 / \mathrm{n}}$ - law of the following form using the lsqcurvefit function of the MatLab package.

$\mu(x)=\mu_{o}+1.0857 b_{n}\left(\frac{x}{x_{e}}\right)^{1 / n}$

Where $b_{n} \cong 2 n-0.327$

$$
\mu_{o}=\mu_{e}-1.0857 b_{n}
$$

For this new value of $\mathrm{x}_{\mathrm{e}}$ a new range of radius is updated as $0.1 x_{e}^{1 / n} \leq r^{1 / n} \leq 1.5 x_{e}^{1 / n}$ then the procedure of the fitting is repeated to updated the value of $x_{e}$ and then the range of radius until converged $x_{e}$ was obtained.

For each galaxy of the adopted sample, the deviation profiles $\delta \mu(\mathrm{x})$ from the best fitting Sersic's model and the mean deviation $<\delta \mu>$ were determined using the following two equations:

$$
\begin{aligned}
& \delta \mu(x)=\mu(x)-\mu_{s}(x) \\
& <\delta \mu>=\frac{1}{\sqrt{N}}\left[\sum_{i}^{N}\left(\mu\left(x_{i}\right)-\mu_{s}\left(x_{i}\right)\right)^{2}\right]^{\frac{1}{2}}
\end{aligned}
$$

Where $\mu(\mathrm{x})$ is the observed surface brightness profiles, $\mu_{\mathrm{s}}(\mathrm{x})$ is the best fitting Sersic 's law to the surface brightness profiles, and $\mathrm{N}$ is the total number of the data points within the radial range

\section{Results and Discussion:}

The best fitting of the $\mathrm{r}^{1 / \mathrm{n}}$ - law to the 46 surface brightness profiles of the adopted sample of elliptical galaxies (see figure(1) which shows the distribution of their absolute magnitude) were done for the intermediate radial range $0.1 \leq\left(\frac{x}{x_{e}}\right) \leq 1.5$. The mean deviations $<\delta \mu>$ for $85 \%$ of the galaxies were found to be less than (0.03) mag. $\operatorname{arcsec}^{-2}$ see figure (2). Only six of the galaxies from the sample (NGC 4926, Coma\#152, NGC 4881, NGC 3091, NGC 3305 and NGC 3308) showed that the mean deviations $<\delta \mu>$ greater than (0.04) mag $\operatorname{arcsec}^{-2}$.

Figure(3) shows the observed profiles and their best fitting for the two galaxies (NGC 4926, NGC 2986), also the figure shows the deviations from the $r^{1 / n}-$ law, for the galaxy NGC 4926 it's deviation profile shows a negative deviation (i.e. the surface brightness of the 
galaxy brighter than $\mathrm{r}^{1 / \mathrm{n}}$ - law) at the inner parts of galaxy, the maximum negative deviation (bottom of a dip) appeared at the intermediate reduced radius $\left(\frac{x}{x_{e}}\right)^{1 / n}=r_{p-}^{\prime}=0.727$, then the profile shows a positive deviation (i.e. the surface brightness of the galaxy fainter than the $r^{1 / n}-$ law) at the outer parts of the galaxy, the maximum positive deviation (top of the hump) appeared at the reduce radius $r_{p^{+}}^{\prime}=0.886$. While for the galaxy NGC 2986, it's deviation profile shows a positive deviation for inner parts of the galaxy then a negative deviation for the outer parts of the galaxy.

The deviation profiles of $57 \%$ of the galaxies show a negative deviation at small radii then positive deviation at large radii, while $43 \%$ of the galaxies show a positive deviation at small radii then negative deviation for large radii. Figure (4) shows the position of the top of the hump of the deviation profiles for the sample which is found at the reduced radius $r_{p^{+}}^{\prime}=0.96$, while figure (5) shows the bottom of the dip of the deviation profiles for the sample of galaxies which is found to be at the reduced radius $r_{p-}^{\prime}=0.83$.

The crossing points $\left(\mathrm{r}_{\mathrm{c}}^{\prime}\right)$ (i.e. the points at which the deviations change their signs) have been found for each galaxy see for example figure (6). The first crossing points, the second and the third, for all galaxies of the sample found to be around the reduced radius $r^{\prime} \cong 0.65$, $r^{\prime}{ }^{\prime} \cong 0.83$ and $r^{\prime}{ }^{\prime} \cong 1.0$ see figure $(7,8$, and 9$)$.

The derived parameters of the best fitting profile and of the deviation profiles are listed in table (1): Column 1- 12, name of galaxy, absolute magnitude $\left(\mathrm{M}_{\mathrm{B}}\right)$, mean deviation $<\delta \mu>$, effective radius of the intermediate range radii $\left(\mathrm{x}_{\mathrm{e}}\right)$, shape parameter $(\mathrm{n})$, reduce radius of the first crossing point $\left(\mathrm{r}_{\mathrm{C} 1}^{\prime}\right)$, reduced radius of the second crossing point $\left(\mathrm{r}_{\mathrm{C} 2}\right)$, reduced radius of the third crossing point $\left(\mathrm{r}_{\mathrm{C} 3}^{\prime}\right)$, the position of the top of the hump $\left(\mathrm{r}_{\mathrm{p}+}\right)$, the maximum positive deviation $\left(\mathrm{p}_{+}\right)$, the position of the bottom of the dip $\left(\mathrm{r}_{\mathrm{p}-}^{\prime}\right)$, and the last column is maximum negative deviation (p.).

\section{Conclution:}

The average of the mean deviation for all the galaxies of the sample is found to be $0.02 \mathrm{mag} \operatorname{arcsec}^{-2}$, see figure (2), these values are smaller than what have been found by Burkert (1993) and Younis (2000 a,b), therefore these results indicate that the Sersic's law fit the surface brightness distribution for the adopted radial range better than the $\mathrm{r}^{1 / 4}$ law.

The deviation profiles of all the galaxies of our sample (except the galaxy NGC 4839) have an excess light than Sersic's law, the bottom of a dip of the deviation profiles found to be around the reduce radius $r_{p-}^{\prime} \cong 0.83$ (see figure 5) this value is in agreement with Burket (1993) and Younis (2000 a,b). 
The results also show that the observed surface brightness of all the galaxies have a dip in their lights comparing to Sersic' law around the reduced radius (i.e the tops of the humps of the deviation profiles) $r_{p^{+}}^{\prime} \cong 0.96$. See figure (4) these result also in agreement with previous studies.

The deviation between the observation profile of the galaxies and their adopted models of Sersic's law has a regular increase or decrease in the surface brightness compared to Sersic's law (see for example figure 3) this mean that these deviations are a real features in the galaxies and not observation errors, besides that the crossing points and the tops of the humps and the bottoms of a dips of the deviation profiles have been found to be converge around certain values so the details studies of such deviations might gives a useful information to the theories that concern the formation of elliptical galaxies see figures $(7,8,9)$

\section{References:}

1) Burkert, A., (1993) "Do Elliptical Galaxies Have $r^{1 / 4}$ Brightness Profiles?". Astro. \& Astrophys., 278, 23-28.

2) Caon, N., Capaccioli, M., D'Onofrio, M., (1993) "On The Shape Of The Light Profiles Of Early-Type Galaxies". Mon. Not. Roy. Astro. Soc., 256, 1013.

3) Capaccioli M., Cappellaro E., Held E. V. and Vietri M., (1993) "Deep kinematics and dynamics of edge-on SO galaxies I.NGC 3115" Astron. Astrophys., 274, 69.

4) Cellone S., (1999) "Surface Brightness profiles of dwarf galaxies in the NGC 5044 Group: A critical revision of the luminosity - shape relation as a distance indicator" Astron. Astrophys., 345,401-413.

5) de Vaucouleurs, G., (1948) "Recherches sur les Nebuleuses Extragalactiques". Ann. Astrophys., 11, 247.

6) Jorgensen, I., Franx, M., Kjaergaard, P., (1992) "CCD surface photometry for E and SO galaxies in the Coma cluster", Astro. \& Astrophys. Suppl., 95, 489.

7) Okamura, S., (1988) "Surface Photometry Of Galaxies". Publ. Astro. Soc. Paci., 100, 524-544.

8) Sersic, J. L., (1968) Atlas de Galaxias Australes (Cordoba: Observatorio Astronomico, Universidad de Cordoba).

9) Trujillo, Graham, A. W., Caon N., (2001) "On the estimation of galaxy structural parameters: the sersic model". Mon. Not. R. Astron. Soc., 326, 869-876.

10) Younis, S. M., (2000a) "Systematic Deviations From $r^{1 / 4}$ ed Vaucouleurs Law In Elliptical Galaxies". J. Edu. Sci., 41.

11) Younis, S. M., (2000b) "Surface Brightness Profiles In Early-Type Galaxies: A Study Of The Small Deviations From The $r^{1 / 4}$ Law". J. Edu. Sci., 42, 77. 
Deviations of the Light Distribution from the Sersic's model in Elliptical ...

Table (1)

\begin{tabular}{|c|c|c|c|c|c|c|c|c|c|c|c|}
\hline galaxy & $\begin{array}{c}-\mathbf{M}_{\mathbf{B}}{ }^{*} \\
\mathrm{mag} .\end{array}$ & $\begin{array}{c}<\delta \mu>> \\
\text { mag } \\
\operatorname{arcsec}^{-2}\end{array}$ & $\begin{array}{c}\mathbf{X}_{\mathbf{e}} \\
\operatorname{arcsec}\end{array}$ & n & $\mathbf{r}_{\mathrm{C} 1}^{\prime}$ & $\mathbf{r}_{\mathrm{C} 2}^{\prime}$ & $\mathbf{r}_{\mathrm{C3}}^{\prime}$ & $\mathbf{r}^{\prime} \mathbf{P +}$ & $\underset{\underset{\operatorname{magsec}^{-2}}{\mathbf{p}_{+}}}{ }$ & $\mathbf{r}^{\prime} \mathbf{P}$ & $\begin{array}{c}\text { p. } \\
\text { mag } \\
\text { arcsec }\end{array}$ \\
\hline$\overline{\mathrm{GC4839}}$ & 21.91 & 0.021 & 5.76 & 069 & 701 & 7779 & $\overline{D 00}$ & 731 & .036 & 000 & 000 \\
\hline & 1.19 & 446 & .17 & 793 & 677 & $\overline{8003}$ & 970 & 886 & (2) & 2273 & \\
\hline IC3959 & 0.06 & $\overline{24}$ & 557 & 590 & 659 & 8565 & 095 & 949 & 018 & $\overline{7337}$ & \\
\hline IC3957 & .52 & 0.014 & 465 & 395 & 827 & 8746 & 979 & 030 & 017 & .0061 & $\overline{0.0243}$ \\
\hline NGC486 & 0.50 & 0.014 & 119 & 2.466 & .568 & 0.7896 & .044 & .937 & .006 & 0.6364 & 0.0173 \\
\hline 106 & \begin{tabular}{|l|l|}
18.79 \\
\end{tabular} & 0.016 & 289 & 2.382 & 775 & 0.9466 & .105 & .020 & 017 & 0.8695 & $-\mathbf{- 0 . 0 2 4 8}$ \\
\hline 107 & 9.19 & 0.023 & 3.876 & 1.905 & .583 & 1.0179 & 000 & .160 & 016 & .8588 & 0.0203 \\
\hline 906 & .17 & 0.020 & 074 & 3.203 & .615 & $\mathbf{0 . 7 9 0 1}$ & 013 & 902 & 020 & 8899 & $\overline{0273}$ \\
\hline NGC & .97 & & 149 & 2.264 & 629 & $\overline{8186}$ & 013 & 415 & & 005 & \\
\hline 125 & 18.72 & 0. & 814 & 44.059 & 871 & $\overline{9622}$ & 027 & 97 & & & \\
\hline 12 & $\overline{9.01}$ & 0.017 & $\overline{244}$ & 1.566 & 567 & 8620 & 243 & 342 & 32 & $\overline{175}$ & 238 \\
\hline 127 & 8.51 & $\overline{01}$ & 177 & 017 & $\overline{800}$ & $\overline{9524}$ & $\overline{000}$ & $\overline{004}$ & $\overline{029}$ & $7 \overline{711}$ & $\overline{222}$ \\
\hline 128 & 8.70 & 7 & 620 & 2.834 & 818 & 1.0009 & .172 & 226 & 025 & 1088 & 309 \\
\hline NGC & 2.59 & .010 & 3.75 & 2.541 & 718 & 0.7892 & .899 & .864 & 2 & 195 & 129 \\
\hline$\overline{71}$ & 9.68 & & $\overline{724}$ & 2.152 & 687 & & 037 & 343 & & & \\
\hline 131 & $\overline{0.19}$ & 0.038 & $\overline{1.44}$ & $\overline{c 6.248}$ & $\overline{c 698}$ & $\overline{228}$ & $\overline{872}$ & $\overline{994}$ & $1 / 2$ & $\overline{210}$ & $\overline{646}$ \\
\hline & 82 & & & 3.843 & 30 & & & 20 & & & \\
\hline 135 & 59 & & & 2.894 & 96 & & $\overline{97}$ & 24 & & 579 & \\
\hline 13 & 64 & & $\overline{70}$ & 2.151 & $\overline{837}$ & $\begin{array}{ll}1.1598 \\
\end{array}$ & $\overline{000}$ & $\overline{258}$ & & $\overline{079}$ & \\
\hline & .16 & & & 3.675 & 36 & 8022 & .010 & 077 & & 216 & \\
\hline NGC & 2.73 & 4 & .25 & 3.786 & $\overline{775}$ & " 0.9448 & $\overline{0000}$ & 918 & $\overline{017}$ & $\overline{555}$ & $\overline{342}$ \\
\hline & $\overline{0.50}$ & 12 & 34 & $\overline{290}$ & $\overline{679}$ & $\overline{7672}$ & $\overline{\mathbf{8 8 7}}$ & $\overline{011}$ & $\overline{\mathbf{0 2 3}}$ & $\overline{457}$ & $\overline{143}$ \\
\hline NG & 0.44 & 20 & 47 & 4.600 & 84 & 13 & 030 & .075 & & $\overline{994}$ & 375 \\
\hline 152 & 19.98 & 0.047 & $\overline{727}$ & 3.969 & .643 & 0.8340 & $\overline{\mathbf{0 0 0}}$ & 1.035 & & $\overline{405}$ & $\overline{\mathbf{7 6 7}}$ \\
\hline & 16 & & & $\mathbf{3 . 0 5 7}$ & 03 & 224 & 056 & 01 & & 880 & \\
\hline & .43 & 11 & 44 & 4.581 & 630 & 880 & 0000 & 778 & & 155 & \\
\hline & .52 & & 313 & 2.453 & 551 & 560 & .998 & .878 & 2 & 193 & \\
\hline & 20.25 & & & 1.642 & & & 000 & 40 & & & \\
\hline & 20.76 & & & 1.253 & & & & & & & \\
\hline & & & & & & & & & & & \\
\hline & & & & 7 & & & & 921 & & & \\
\hline & & & & 3 & 51 & $\overline{76}$ & $\overline{36}$ & 928 & & & \\
\hline NGC4 & 22.30 & 0.020 & 38.39 & 7.240 & ב.677 & " 0.7866 & $\overline{0.886}$ & $\overline{0.842}$ & $\overline{0.030}$ & 281 & $\overline{213}$ \\
\hline & $\overline{0.99}$ & & & .704 & 709 & & 856 & 817 & 036 & 482 & -0.0326 \\
\hline IC2623 & 19.24 & 0.004 & 3.535 & 2.010 & 0.624 & $\mathbf{0 . 7 8 3 5}$ & 0.953 & 0.691 & 0.006 & 0.8762 & $-\mathbf{- 0 . 0 0 5 5}$ \\
\hline & 20.55 & & & 3.974 & & & 036 & 759 & & 412 & 0382 \\
\hline$\overline{986}$ & 20.55 & 0.015 & 41.91 & 2.978 & 0.697 & 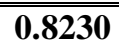 & 0.962 & 0.756 & .024 & $\overline{0.8869}$ & -0.0203 \\
\hline GC3091 & 21.37 & 0.046 & 9 & 679 & 96 & 36 & 909 & 231 & 1 & $\overline{884}$ & 629 \\
\hline 268 & 20.66 & 0.022 & 72.66 & 4.322 & 787 & $\begin{array}{c}\mathbf{0 . 8 7 8 3} \\
\end{array}$ & 0000 & 0.847 & 0.030 & 0.9253 & 0439 \\
\hline & $\overline{144}$ & & & 0 & 46 & 14 & 4 & 928 & 5 & 81 & $\overline{686}$ \\
\hline & & & & & & & 69 & 26 & & & \\
\hline & & & & & & & & & & & \\
\hline NGC4373 & 21.94 & 0.015 & 68.05 & 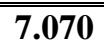 & 0.767 & 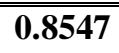 & 0.942 & 0.808 & 0.023 & $\overline{c 0.8998}$ & -0.0251 \\
\hline & 21.17 & & & 4.310 & 685 & & 1.035 & 096 & 37 & 182 & $-\mathbf{0 . 0 2 7 9}$ \\
\hline & 20.48 & & & $\begin{array}{l}8.781 \\
\end{array}$ & $\begin{array}{l}0.783 \\
\end{array}$ & & $\begin{array}{l}0.903 \\
\end{array}$ & $\begin{array}{l}0.935 \\
\end{array}$ & & & $\overline{314}$ \\
\hline GC5903 & .06 & 0.04 & 09 & 7 & 0.686 & 0.8241 & 961 & 0.752 & 0.037 & 0.8989 & -0.0433 \\
\hline
\end{tabular}

* Jorgensen et al 1992 


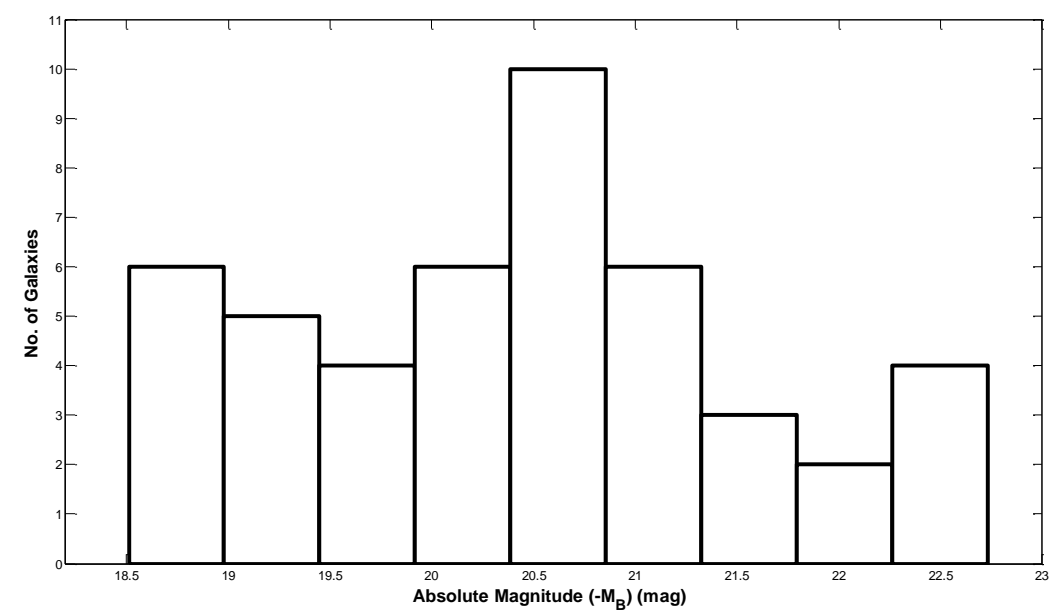

Figure(1): The distribution of the elliptical galaxies as a function of their absolute magnitude

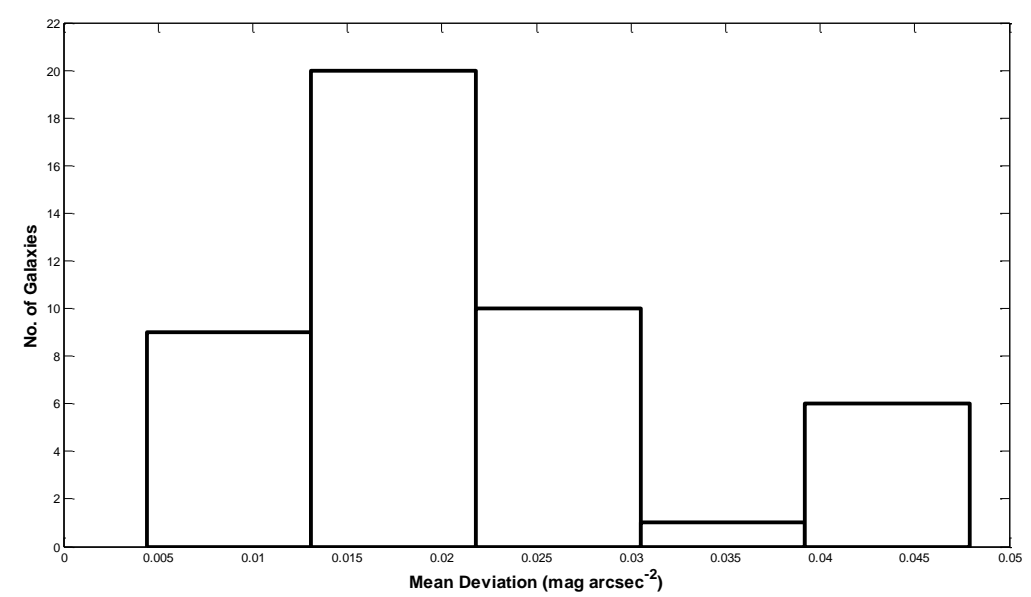

Figure(2): the number of Elliptical galaxies is shown as a function of their mean deviations $\langle\delta \mu>$
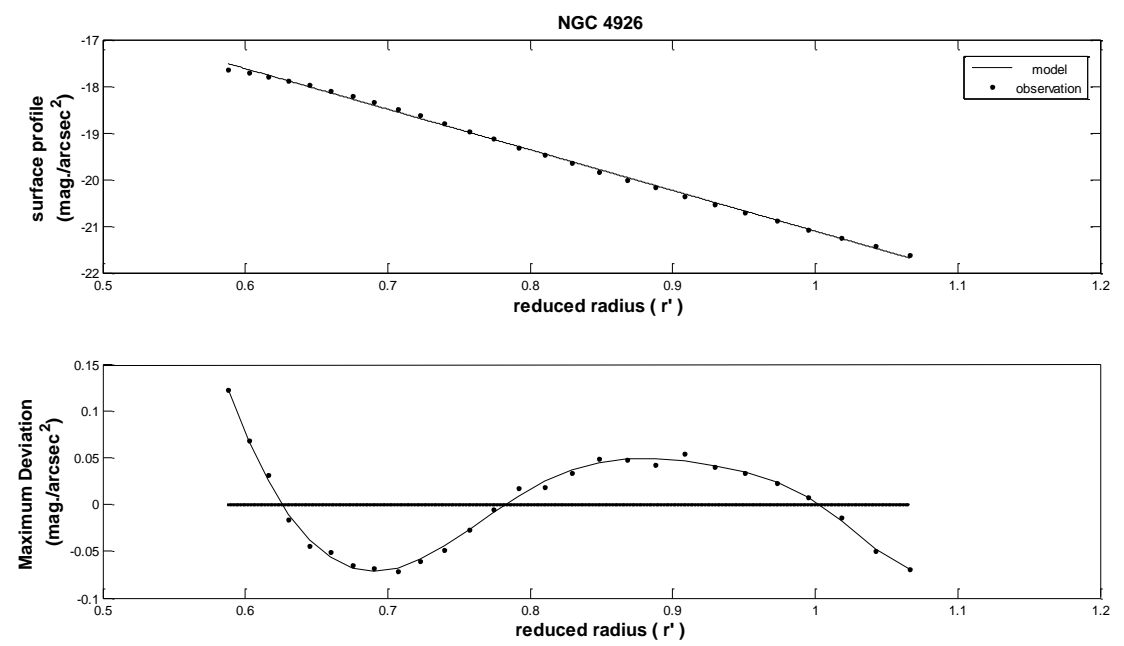

Figure(3)a 
Deviations of the Light Distribution from the Sersic's model in Elliptical ...
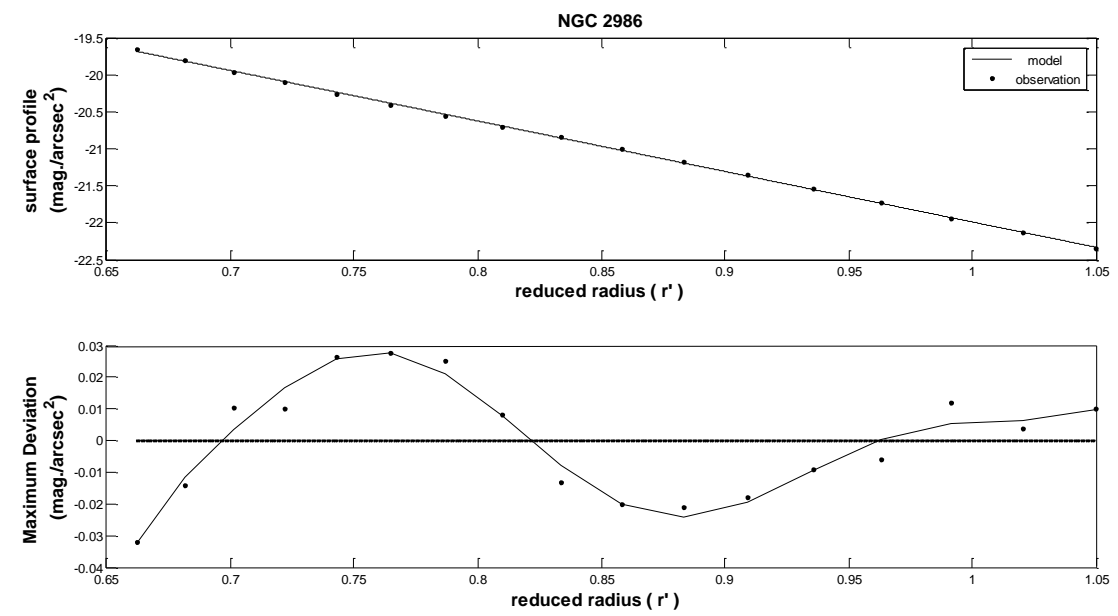

Figure(3)b

Figure(3): The radial deviation profiles for the elliptical galaxies NGC4926 and NGC 2986 versus $r$ as an example of the sample

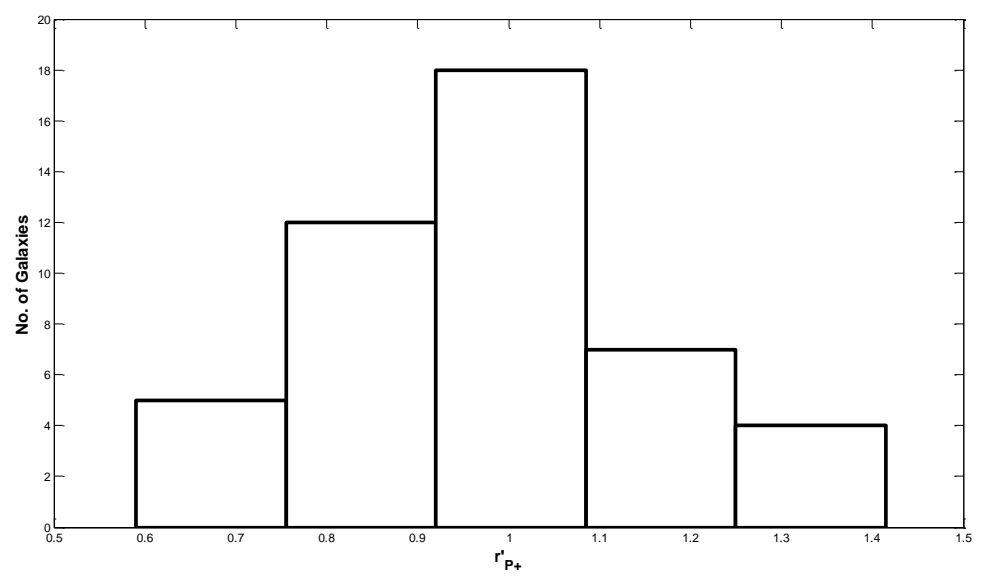

Figure(4): The reduced radius of the maximum positive deviation (top of the hump) $\mathbf{r}_{P+}^{\prime}$

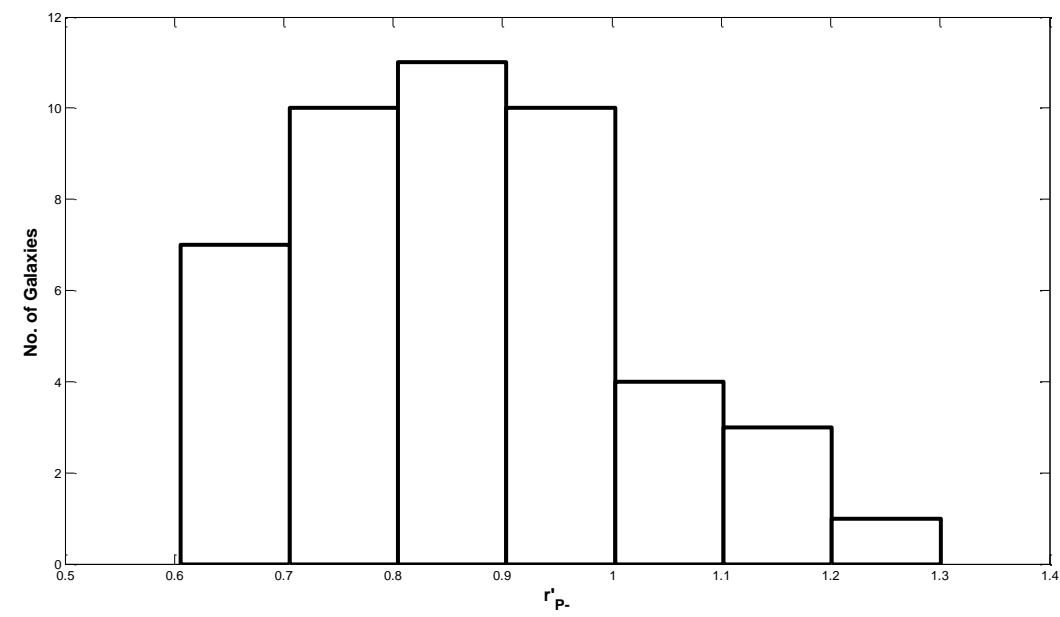

Figure(5): The reduced radius of the maximum negative deviation (Lower point) $\mathbf{r}_{\mathbf{P}}^{\prime}$ 


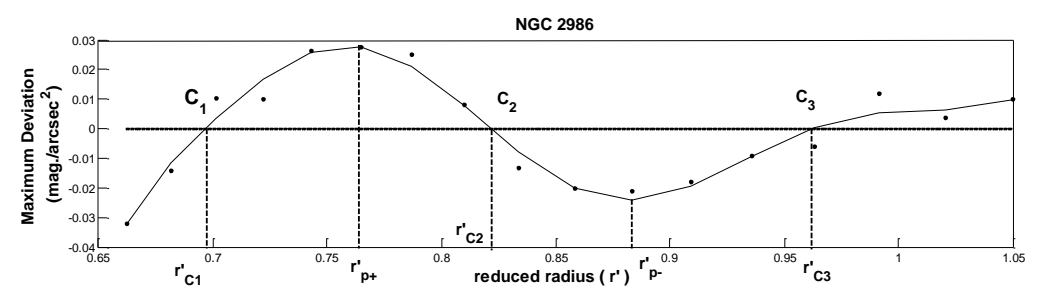

Figure(6): Represent the adopted parameters of the deviation profile: $c_{1}, c_{2}$ and $c_{3}$ are the crossing points: $\mathbf{r}_{\mathrm{C}_{1}}, \mathbf{r}_{\mathrm{C} 2}$ and $\mathbf{r}_{\mathrm{C} 3}$ are the reduced radius of the crossing points: $\mathbf{r}_{\mathrm{P}+}^{\prime}$ is the the reduced radius for the top of the hump of the deviation where $\mathbf{r}^{\prime}{ }_{\mathrm{p}}$ is the reduced radius for the bottom of the dip of the deviation.

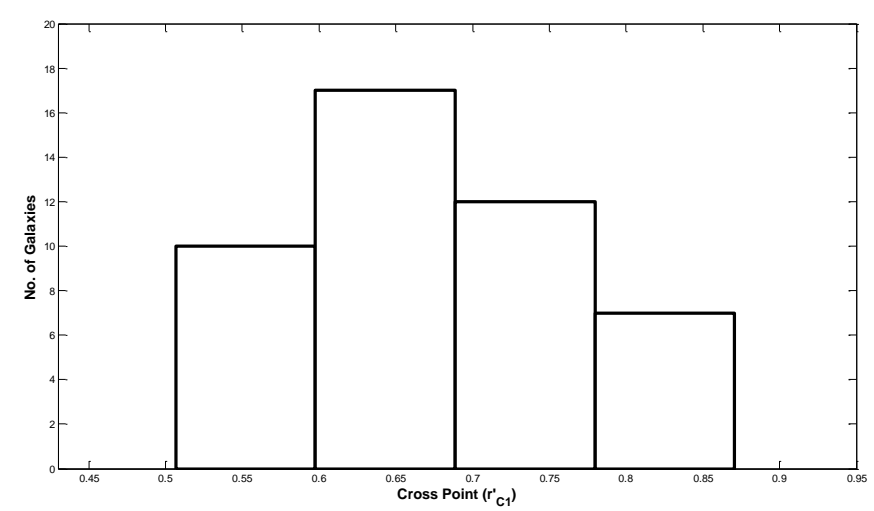

Figure(7): The crossing point at $\mathbf{r}_{\mathrm{C} 1}^{\prime}$

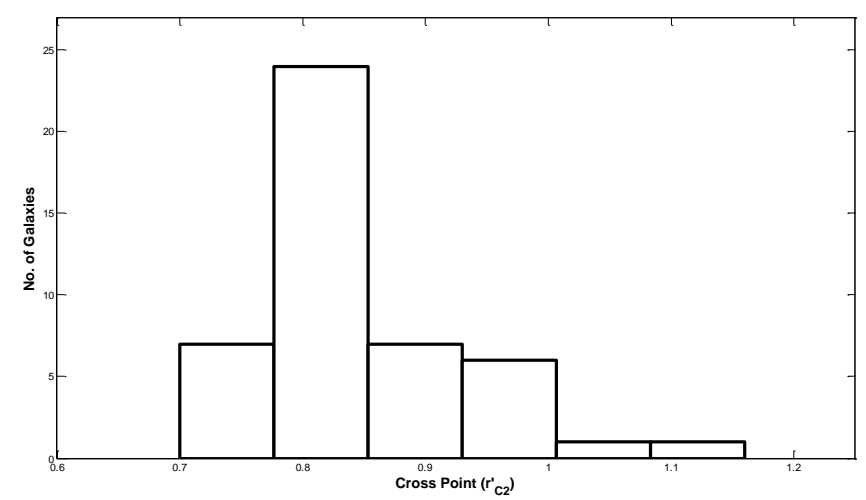

Figure(8): The crossing point $\left(\mathbf{r}^{\prime}{ }_{2}\right)$

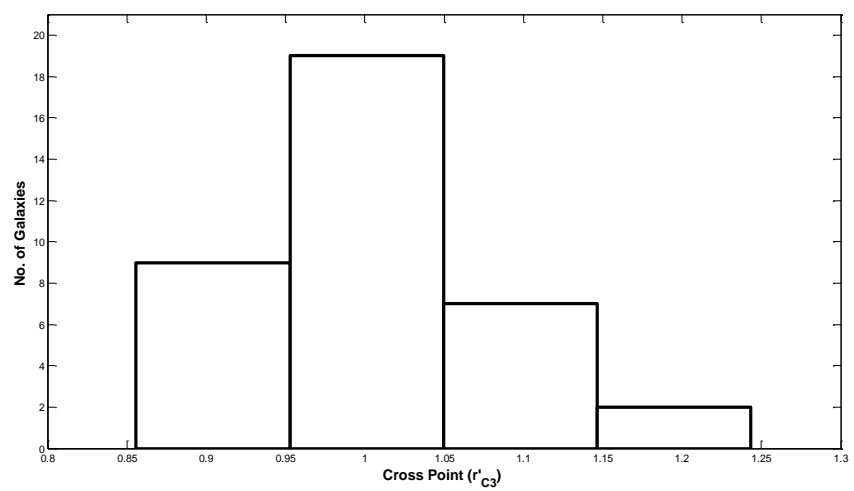

Figure(9): The crossing point $\left(\mathbf{r}^{\prime}{ }^{\prime}\right)$ 